\title{
Eficiencia de las redes atencionales en personas con diabetes mellitus tipo 1: Estudio piloto
}

Noviembre 2020, Vol.

$12, N^{\circ} 3,82-91$

revistas.unc.edu.ar/inde x.php/racc

Manríquez-Calderón, Sarahi del Carmen *, a; Padrós-Blázquez, Ferrán a y Villuendas-González, Erwin Rogelio ${ }^{\mathrm{a}}$

\section{Artículo Original}

\section{Resumen}

Numerosos estudios han documentado que los pacientes con diabetes mellitus tipo 1 (DMT1) son susceptibles de padecer complicaciones estructurales, metabólicas y funcionales en el sistema nervioso central. Con el fin de comparar la eficiencia de las redes atencionales entre pacientes con DMT1 y personas sanas, 5 pacientes con DMT1 y 5 personas sanas respondieron el Test de Redes Atencionales. Se analizó la eficiencia de las redes atencionales: alerta, orientación y control ejecutivo en ambos grupos, así como la correlación entre la eficiencia de estas redes y los niveles de glucosa en sangre. Aunque no se observaron diferencias significativas en la eficiencia de las redes entre los grupos, sí se observó una relación entre la glucemia y la eficiencia de la red de control, así como también en la influencia de la red de control sobre la red de orientación.

\section{Abstract}

Efficiency of attentional networks in people with type 1 diabetes mellitus: Pilot study. Many studies have documented that patients with type 1 diabetes mellitus (T1DM) are susceptible to structural, metabolic and functional complications involving the central nervous system. To compare the efficiency of attentional networks in T1DM and healthy adults, 5 patients with T1DM and 5 healthy people answered the Attentional Network Test. We analyzed the efficiency of attentional networks: alerting, orienting and executive control in both groups, and the correlation between the efficiency of these networks and blood glucose levels. Although no significant differences in the efficiency of networks between groups were observed, a relation between glycaemia and efficiency of the control network was observed, as well as the influence of the control network on the orientation network.
Tabla de Contenido

\begin{tabular}{ll}
\hline Introducción & 82 \\
Método & \\
Participantes & 83 \\
Instrumentos & 84 \\
Procedimiento & 84 \\
Análisis de los & 85 \\
datos & \\
Resultados & 85 \\
Discusión y & 87 \\
Conclusiones & \\
Agradecimientos & 89 \\
Referencias & 89
\end{tabular}

Palabras clave:

diabetes mellitus tipo 1, redes atencionales,

glucemia, cognición.

Recibido el 23 de enero de 2018; Aceptado el 25 glycaemia, cognition.

Editaron este artículo: Eliana Rueti, Mariana Bentosela, Paula Abate, Gabriela Raynaudo y María Victoria Ortiz

\section{Introducción}

La Organización Mundial de la Salud reportó que en el año 2014 existían 422 millones de adultos con diabetes mellitus (DM) en el mundo, lo que corresponde a 1 de cada 11 personas (Organización Mundial de la Salud, 2016); además, señaló que 1.5 millones de personas mueren cada año a causa de esta enfermedad. La diabetes mellitus tipo 1 (DMT1) es una enfermedad metabólica crónica que se origina cuando en el páncreas existe un fallo total de la secreción de insulina y su etiología suele ser autoinmune. El efecto de la DMT1 no controlada es la hiperglucemia, que con el tiempo origina diferentes complicaciones patológicas entre las que se encuentran cambios estructurales, metabólicos y funcionales en el sistema nervioso central. Datos ofrecidos por diversas investigaciones muestran evidencia clínica de deficiencias cognitivas encontradas en niños, jóvenes, adultos, así como en personas de la tercera edad con DMT1 en comparación con sujetos control (Brands et al., 2006; Ferguson et

\footnotetext{
a Universidad Michoacana de San Nicolás de Hidalgo, Facultad de Psicología, Morelia, Michoacán, México.

*Enviar correspondencia a: Manríquez-Calderón, S. E-mail: sarahimzcn@gmail.com
} 
Manríquez-Calderón, S., et al. / RACC, 2020, Vol. 12, N³, 82-91

al., 2005; Kirchhoff, Jundt, Doty, \& Hershey, 2017; Li, Huang, \& Gao, 2017; Lin, Northam, Werther, \& Cameron, 2015; Marzelli et al., 2014; Semenkovich et al., 2016). Aunque todas las alteraciones cognitivas encontradas en estos pacientes van de leves a moderadas, podrían comprometer el desarrollo de diversas tareas cognitivas, sobre todo en aquellas de mayor complejidad (Brands, Biessels, De Haan, Kappelle, \& Kessels, 2005; Naguib, Kulinskaya, Lomax, \& Garralda, 2009).

La mayoría de los estudios de la literatura señalan que los déficits encontrados, a partir de la evaluación con múltiples pruebas, se relacionan con la hipoglucemia, la hiperglucemia 0 la presencia de ambos extremos glucémicos (Asvold, Sand, Hestad, \& Bjorgaas, 2010; BadeWhite \& Obrzut, 2009; Cato \& Hershey, 2016; Ferguson et al., 2003; Hannonen, Tupola, Ahonen, \& Riikonen, 2003; Kodl \& Seaquist, 2008; Lin, Northam, Rankins, Werther, \& Cameron, 2010; Marzelli et al., 2014; Patiño-Fernández et al., 2010; Rodrigues, de Castro, Schamber, \& Bazotte, 2014; Ryan, Klein, Lee, Cruickshanks, \& Klein, 2016).

Una de las funciones cognitivas que se ha visto comprometida en pacientes con DMT1 es la atención (Kodl \& Seaquist, 2008). Gaudieri, Chen, Greer y Holmes (2008), analizaron datos de 19 estudios encontrando que las alteraciones en atención están consistentemente relacionadas con la DMT1. La mayoría de estos hallazgos en el funcionamiento atencional se relacionan a la presencia frecuente de episodios de hipoglucemia e hiperglucemia (Bade-White \& Obrzut, 2009; Hannonen et al., 2003) y al inicio temprano de la enfermedad (Ferguson et al., 2003; Ferguson et al., 2005; Marzelli et al., 2014).

Pese a que en gran parte de las investigaciones no se tienen datos precisos sobre las alteraciones presentes en los diferentes componentes de la atención, algunos estudios sugieren que las alteraciones atencionales en pacientes con DMT1 se encuentran específicamente en atención sostenida (Brands et al., 2005; Ferguson et al., 2005; Lin et al., 2010; Naguib et al., 2009) y dividida (Lin et al., 2010). En un metanálisis realizado por McCrimmon, Ryan y Frier (2012), se muestra que la DMT1 tiene un efecto sobre diferentes dominios cognitivos. Dentro de estos dominios, la atención es una de las funciones cognitivas que resulta mayormente alterada, específicamente la atención visual y sostenida.

Como las células de todo el cuerpo son especialmente susceptibles a las alteraciones de los niveles de glucosa en sangre, entre ellas las células presentes en el sistema nervioso central, cabría esperar que el funcionamiento global del cerebro se vea particularmente afectado. Estos cambios podrían compensarse de alguna manera a través de diversos mecanismos, como el uso de redes más extendidas o un mayor esfuerzo ante tareas exigentes. Si bien la eficiencia para responder puede ser prácticamente normal, el registro computarizado de tiempos de respuesta podría revelar diferencias sutiles entre el desempeño de personas con DMT1 comparadas con personas sanas. Estas diferencias podrían observarse tanto en la eficiencia de cada una de las redes como en su interacción, la cual generalmente se estudia a partir de correlaciones entre eficiencia de las redes, pero no a través de índices de interacción (e.g. MacLeod et al., 2010). Con todo lo mencionado anteriormente, el objetivo de la presente investigación fue comparar la eficiencia de las redes atencionales en un grupo de personas con DMT1 versus controles sanos, durante la ejecución de tareas atencionales, y estudiar la relación entre los niveles de glucosa y dicha eficiencia.

\section{Método}

\section{Participantes}

Los participantes fueron seleccionados a través de un muestreo intencional por cumplimiento de los criterios de inclusión: edad entre 18 y 40 años, diagnosticados con DMT1 (insulinodependientes), estimación de $\mathrm{Cl}$ no menor de 90 (a través de la aplicación de la Escala Wechsler de Inteligencia para Adultos abreviado dual), evolución de la enfermedad mayor a 5 años, escolaridad de 9 años o superior, visión normal o corregida, que asisten al Hospital General de Morelia "Dr. Miguel Silva". El grupo control se conformó con personas sanas equiparadas en edad, sexo y nivel educativo con el grupo de pacientes. Ninguno de los participantes fue eliminado del estudio. La muestra final estuvo formada por 5 participantes con DMT1 y 5 participantes sanos (ver tabla 1). 
Manríquez-Calderón, S., et al. / RACC, 2020, Vol. 12, N³, 82-91

Tabla 1.

Datos sociodemográficos de los participantes

\begin{tabular}{ccccc}
\hline Grupo & Sexo & Edad & $\begin{array}{c}\text { Años de } \\
\text { escolaridad }\end{array}$ & Cociente Intelectual (CI) \\
\hline Experimental & Mujer & 22 & 13 & 91 \\
1 & Mujer & 16 & 10 & 91 \\
2 & Mujer & 16 & 10 & 91 \\
3 & Mujer & 19 & 12 & 97 \\
4 & Hombre & 33 & 17 & 97 \\
5 & & 21.2 & 12.4 & 93.4 \\
Promedio & & & & 91 \\
Control & Mujer & 18 & 12 & 103 \\
1 & Mujer & 18 & 12 & 91 \\
2 & Hombre & 34 & 17 & 103 \\
3 & Mujer & 20 & 12 & 94 \\
4 & Mujer & 19 & 12 & 96.4 \\
\hline 5 & & 21.8 & 13 & \\
\hline
\end{tabular}

\section{Instrumentos}

Se evaluó la eficiencia atencional con la aplicación del Attentional Network Test (ANT) (Fan, McCandliss, Sommer, Raz, \& Posner, 2002), en la versión de Wang et al. (2015). La versión de Wang et al. (2015) difiere de la original en dos aspectos: la eficiencia de las redes se evalúa en bloques independientes (sin condiciones que se evalúen de manera ortogonal) y los índices de eficiencia e interacción entre redes se calculan a partir de razones entre los tiempos de respuesta a las condiciones asociadas a cada red. Esta organización a modo de bloques disminuye el efecto que la exigencia a una red en un ensayo dado puede tener sobre los ensayos subsecuentes (e.g. McConnell \& Shore, 2011; Wang et al., 2014). El uso de razones para calcular la eficiencia de las redes ayuda a evitar el efecto que deriva de las diferencias individuales en la velocidad de respuesta (e. g. Westlye, Grydeland, Walhovd, \& Fjell, 2010).

El ANT es una prueba informatizada que a través de un paradigma de estímulos flanqueados permite estimar la eficiencia de tres redes (alerta, orientación y control ejecutivo) y su interacción. A lo largo de la prueba se presentan en la pantalla de una computadora flechas que pueden apuntar hacia la derecha o hacia la izquierda. El participante debe identificar la orientación de la flecha central pulsando el botón correspondiente en una caja de respuestas. Los participantes fueron instruidos para responder utilizando el dedo índice de cada mano para pulsar el botón izquierdo y derecho de la caja de respuestas.

Durante la tarea se presentaron seis bloques distintos: tres bloques fueron utilizados para evaluar la eficiencia de las tres redes en forma individual (48 ensayos por bloque) y los otros tres bloques permitieron evaluar la interacción entre las redes (96 ensayos por bloque). Los bloques y los ensayos fueron presentados aleatoriamente con la finalidad de evitar los efectos sistemáticos de la práctica y la fatiga sobre la precisión y tiempos de respuesta. El tiempo de respuesta y la precisión se registraron utilizando una caja de respuesta (Psychology Software Tools Inc., 2012). Todos los bloques fueron precedidos por diez ensayos de práctica para familiarizar a los participantes con la tarea. La tarea fue programada en E-prime 2.0 (Psychology Software Tools Inc., 2012) con los parámetros descritos en Wang et al. (2015) y tuvo una duración aproximada de 30 minutos.

\section{Procedimiento}

El proyecto de investigación fue aprobado por el Comité de Ética de la Facultad de Psicología de la Universidad Michoacana de San Nicolás de Hidalgo y financiado por la Coordinación de Investigación Científica de la Universidad Michoacana de San Nicolás de Hidalgo. Todos los datos de los participantes fueron tratados de forma confidencial y ninguno de sus nombres fue utilizado.

Para la evaluación, primeramente, se explicó 
Manríquez-Calderón, S., et al. / RACC, 2020, Vol. 12, №3, 82-91

a los participantes el motivo del estudio, solicitándoles su participación voluntaria. Posteriormente, se les realizó una entrevista para verificar que cumplieran con los criterios de inclusión. Cuando la muestra fue seleccionada a cada uno de los participantes se le informó con detalle los pormenores del procedimiento y se resolvieron sus dudas. Posteriormente se recabaron las firmas de quienes aceptaron participar en un formato de consentimiento informado.

La experimentación fue realizada en una habitación sonoamortiguada con iluminación eléctrica constante. Además, durante la evaluación les eran colocados a los participantes protectores de oídos para disminuir el ruido del ambiente. Al interior del laboratorio se encuentra un mueble diseñado para que dos personas se coloquen cada una frente a un monitor (ambos conectados a la misma computadora, de manera tal que el evaluador puede ver todo el tiempo lo que el participante está viendo): participante e investigador. Los participantes respondieron la tarea frente a un monitor de 19 pulgadas a una distancia aproximada de $50 \mathrm{~cm}$.

Los participantes asistieron a una sola sesión, en donde al inicio se les realizó una prueba de glucosa capilar. Una vez que eran determinados los niveles de glucosa en sangre, los participantes resolvían el ANT y finalmente se realizaba la determinación individual de $\mathrm{Cl}$ (para verificar el cumplimiento del criterio de inclusión).

\section{Análisis de los datos}

Los datos registrados fueron analizados en $R$ (R Core Team, 2016). Las medianas de los tiempos de reacción en el ANT fueron calculadas tomando en cuenta sólo los ensayos correctos. Para las comparaciones entre grupos se utilizó la prueba no paramétrica de Wilcoxon, y para el análisis de correlaciones se calcularon índices rho de Spearman.

\section{Resultados}

Para calcular la eficiencia de las redes atencionales entre el grupo control y el grupo experimental se obtuvieron las medianas de los tiempos de reacción por condición de cada uno de los participantes. Tanto la eficiencia de las redes atencionales, como la interacción entre ellas fue calculada de acuerdo con el procedimiento descrito por Wang et al. (2015). La tabla 2 muestra las medianas de los índices de eficiencia de las redes atencionales y la interacción entre ellas en ambos grupos. La comparación entre grupo control y grupo experimental se realizó con una prueba no paramétrica de Wilcoxon. Los datos obtenidos no mostraron una diferencia estadísticamente significativa entre la eficiencia de las redes atencionales (ver figura 1): alerta $(W=$ $10, p=.69)$, orientación $(W=9, p=.55)$ y control ejecutivo $(W=8, p=.42)$ del grupo control versus el grupo experimental. En el análisis de la interacción entre las redes atencionales tampoco se observó una diferencia estadísticamente significativa entre el grupo control y el grupo de participantes con DMT1: alerta sobre orientación $(W=8, p=.42)$, orientación sobre alerta $(W=13$, $p=1)$, alerta sobre control ejecutivo $(W=20, p=$ $.15)$, control ejecutivo sobre alerta $(W=12, p=1)$, orientación sobre control ejecutivo $(W=13, p=1)$ y control ejecutivo sobre orientación, $(W=7, p=$ .31).

La correlación entre los índices de eficiencia y la glucemia no fue estadísticamente significativa: alerta ( $r h o=-.40, p=.25)$, orientación $(r h o=-.35$, $p=.31$ ) y control ejecutivo (rho $=-.29, p=.41$ ). La mayoría de los índices de interacción tampoco mostraron una correlación estadísticamente significativa con los niveles de glucosa en sangre: alerta sobre orientación (rho $=.01, p=.97$ ), orientación sobre alerta ( $r h o=-.20, p=.58)$, alerta sobre control ejecutivo ( $r h o=.20, p=.58$ ), control ejecutivo sobre alerta (rho $=-.32, p=.37$ ) y orientación sobre control ejecutivo (rho $=.41, p=$ .23).

La interacción entre la red de control ejecutivo y la orientación ( $r h o=-.68, p=.035$ ) presentó una correlación negativa estadísticamente significativa con los niveles de glucosa presentes en la sangre. Esto implica que en las personas con niveles altos de glucosa el efecto de la red de control ejecutivo impacta en mayor medida al efecto de la red de orientación, en comparación con las personas con niveles normales de glucosa (ver figura 2). 
Manríquez-Calderón, S., et al. / RACC, 2020, Vol. 12, №3, 82-91

Tabla 2.

Medianas de la eficiencia de las redes atencionales y la interacción entre ellas por grupo

\begin{tabular}{lcccccccc}
\hline Grupo & $\mathrm{A}$ & $\mathrm{O}$ & $\mathrm{E}$ & $\mathrm{A}>\mathrm{O}$ & $\mathrm{O}>\mathrm{A}$ & $\mathrm{A}>\mathrm{E}$ & $\mathrm{E}>\mathrm{A}$ & $\mathrm{O}>\mathrm{E}$ \\
\hline Experimental & -0.12 & 0.04 & 0.08 & -0.51 & -0.17 & 0.32 & -0.48 & -1.29 \\
Control & -0.06 & 0.07 & 0.14 & 0.75 & -0.37 & -0.93 & -0.09 & -2.94 \\
\hline
\end{tabular}

Nota. $\mathrm{A}=$ Red de alerta; $\mathrm{O}=$ Red de orientación; $\mathrm{E}=$ Red de control ejecutivo; $\mathrm{X}>\mathrm{Y}=$ Interacción entre las redes
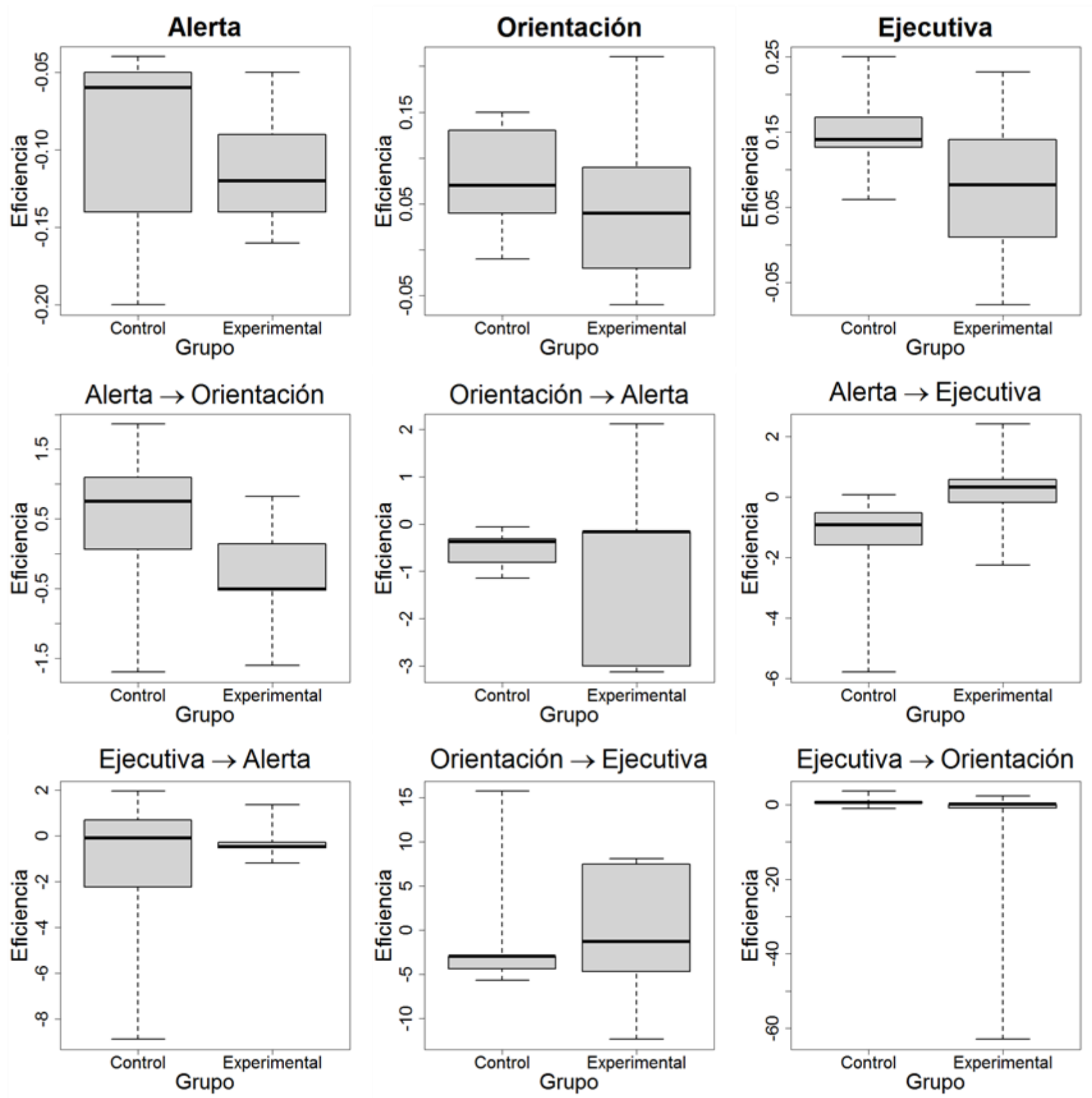

Figura 1. Comparación de los índices de eficiencia de las redes atencionales entre grupos. 
Manríquez-Calderón, S., et al. / RACC, 2020, Vol. 12, N³, 82-91
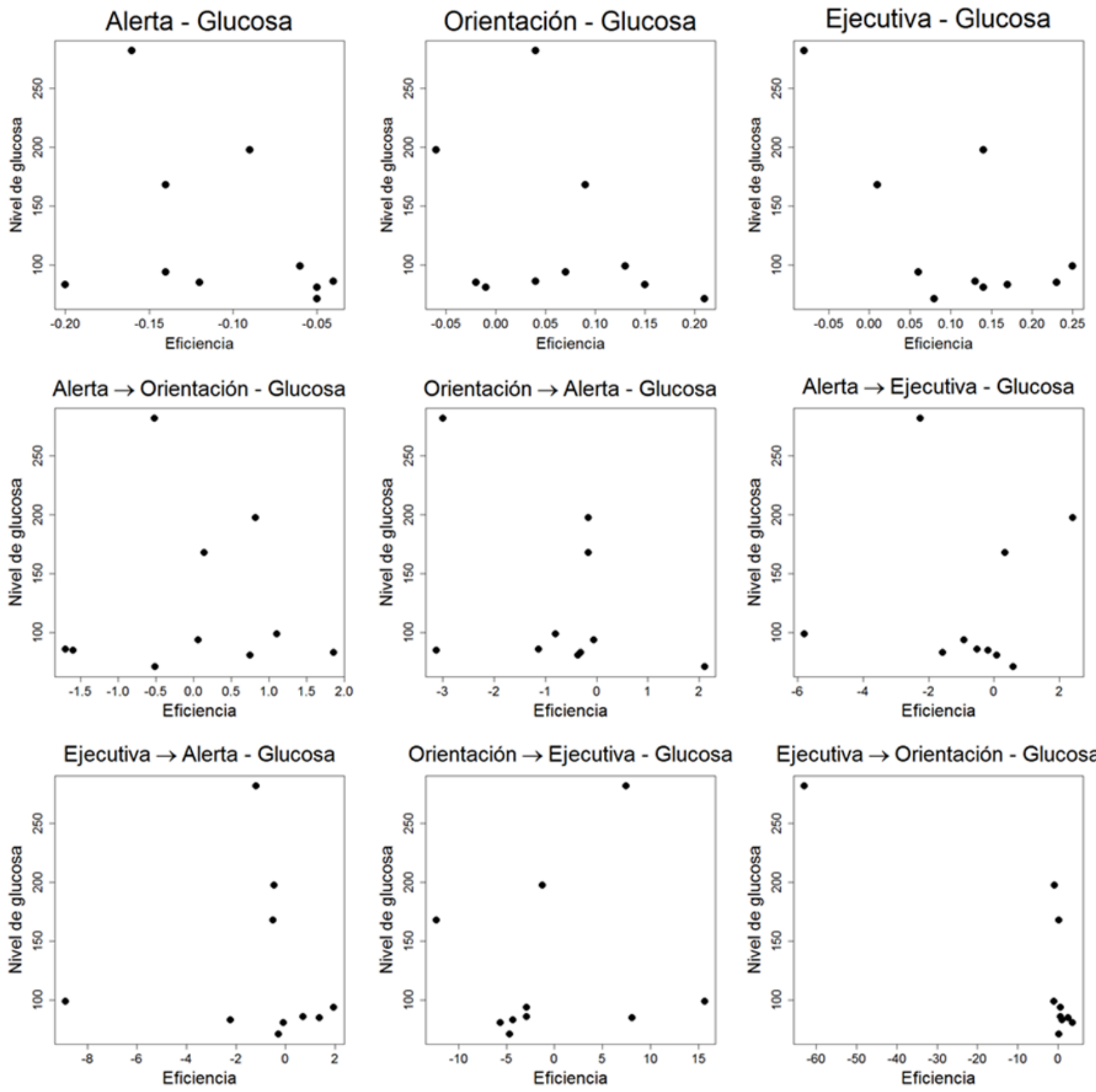

Figura 2. Correlación entre la eficiencia de las redes y los niveles de glucosa en sangre.

\section{Discusión y Conclusiones}

Como ya se ha mencionado, la literatura muestra que los pacientes con DMT1 pueden presentar alteraciones en el funcionamiento cognitivo. Sin embargo, otros estudios reportan alteraciones mínimas, e incluso señalan que los pacientes con DM han mostrado un desempeño similar al de los grupos control. En nuestro caso, los participantes con DMT1 obtuvieron una precisión y tiempos de respuesta similares a los del grupo control durante la ejecución de las tareas atencionales. Los resultados anteriores podrían deberse al tamaño limitado de la muestra, o en caso de que realmente no haya diferencias, esto podría explicarse de dos formas: compensación o limitación de las deficiencias a los episodios de hipo e hiperglucemia. Aunque la DMT1 es una patología que afecta al neurodesarrollo, los pacientes que la padecen mantienen un funcionamiento cognitivo que, si bien puede requerir de mecanismos compensatorios a nivel cerebral, les permite realizar sus actividades diarias de forma regular. Además, la mayoría de las investigaciones 
Manríquez-Calderón, S., et al. / RACC, 2020, Vol. 12, №3, 82-91

reportadas sugieren que las disfunciones cognitivas presentes en la DM pueden explicarse mejor con las alteraciones recurrentes en los niveles de glucosa en sangre o por las distintas condiciones comórbidas que acompañan a dicha enfermedad (Brands et al., 2005).

Asimismo, en nuestro estudio los índices de eficiencia atencional no mostraron diferencias significativas entre los pacientes con DMT1 y los participantes del grupo control. La eficiencia de las redes: alerta, orientación y control ejecutivo, así como la interacción entre ellas mostraron el mismo patrón en los resultados obtenidos. Es importante señalar, que nuestros datos forman parte de un estudio piloto por lo que nuestro tamaño muestral es reducido y los datos deben ser tomados con reserva.

A nivel general, la DM es una condición médica que afecta al sistema nervioso central; no obstante, ha sido reportado que el deterioro cognitivo es más pronunciado en pacientes con diabetes mellitus tipo 2 (DMT2) en comparación con la DMT1 (Brands et al., 2007; Monette, Baird, \& Jackson, 2014). Con ello, se podría esperar que a nivel general los participantes con DMT1 obtuvieran un rendimiento similar al de personas sanas, tal y como sucedió en este estudio en el que los índices de eficiencia de las redes fueron similares en ambos grupos. Las diferencias entre los dos tipos de DM, de acuerdo con lo reportado en la literatura, pueden deberse a diversos factores de riesgo vascular o comorbilidad que son más comunes en la DMT2.

La DMT1 es una enfermedad metabólica que afecta la producción de insulina en el organismo, lo que trae como consecuencia un inadecuado aprovechamiento de la glucosa. La elevación permanente de la glucosa en la sangre (hiperglucemia) se asocia a diferentes patologías entre las que se encuentran las alteraciones cognitivas. Por ello, analizamos la relación entre la eficiencia de las redes atencionales y los niveles de glucosa en sangre. Dicho análisis no mostró una correlación entre los niveles de glucosa en sangre y la eficiencia de las tres redes: alerta, orientación y control ejecutivo, lo que podría indicar que el nivel de glucemia presente en el organismo (específicamente en el cerebro) no tiene un papel determinante en la eficiencia de estas redes. Así, un nivel bajo de glucosa podría acompañarse de la activación de redes distintas a las que se activarían con un nivel normal, y en ambos casos la respuesta a nivel conductual podría ser muy similar, como en los datos reportados por Gallardo-Moreno, GonzálezGarrido, Gudayol-Ferré y Guàrdia-Olmos (2015). Otra razón por la que esta relación pudo no ser significativa estaría en el tamaño reducido de la muestra: índices de correlación con magnitudes similares a los que encontramos implicarían relaciones significativas en muestras de alrededor de 30 participantes.

En contraste, el efecto de la red de control ejecutivo sobre la red de orientación sí se relacionó de forma negativa con los niveles de glucosa. Este hallazgo podría implicar una asociación entre un mayor nivel de glucosa en sangre y una menor eficiencia en la interacción entre la red de control ejecutivo y la orientación (que se reflejaría en un mayor costo en tiempo de respuesta a tareas de orientación cuando la resolución de conflicto ya tiene un costo elevado). La exposición a la hiperglucemia puede tener un efecto perjudicial sobre el cerebro (Ferguson et al., 2003), y los episodios de hiperglucemia son recurrentes en los pacientes con DMT1; la relación hallada en nuestro estudio entre los índices de eficiencia y la glucemia podría ser una consecuencia de ello. Además, la eficiencia de la red de control ejecutivo está relacionada con el adecuado funcionamiento de los lóbulos frontales. Algunas investigaciones han reportado alteraciones en el funcionamiento ejecutivo en pacientes con DMT1 (Gaudieri et al., 2008; Hannonen et al., 2003; Kodl \& Seaquist, 2008; Ohmann et al., 2010) en la planeación, flexibilidad cognitiva y tiempo de reacción (Hannonen et al., 2003; Ohmann et al., 2010). Las diferencias en los tiempos de reacción reportadas por estos autores podrían reflejarse también en una menor eficiencia en la interacción de algunas redes atencionales, como es el caso en nuestro estudio.

Asimismo, se han reportado cambios estructurales en el sistema nervioso central, específicamente alteraciones en las regiones lateral y medial del lóbulo frontal (Marzelli et al., 2014). Otras alteraciones descritas son los cambios observados en el volumen de materia gris y materia blanca en diferentes regiones cerebrales (Marzelli et al., 2014; Wessels et al., 2007). De acuerdo con dichos cambios, el volumen de materia blanca, específicamente se relaciona con la eficiencia de la atención y el funcionamiento ejecutivo. También se ha 
Manríquez-Calderón, S., et al. / RACC, 2020, Vol. 12, №3, 82-91

encontrado que los pacientes con DMT1 con episodios recurrentes de hiperglucemia son especialmente susceptibles de presentar estos cambios estructurales (Ferguson et al., 2003). Comparando nuestros hallazgos con los antes descritos podríamos explicar el efecto que tienen los elevados niveles de glucosa en sangre sobre la eficiencia en la interacción de la red de control ejecutivo obre la orientación.

En resumen y tomando en cuenta que los resultados presentados anteriormente forman parte de un estudio piloto, los hallazgos descritos deben interpretarse tomando en cuenta algunas limitaciones: el tamaño reducido de la muestra, y el efecto de este tamaño reducido sobre el proceso de aleatorización de bloques en la versión del ANT utilizada. Dado que en la versión de Wang et al. (2015) la eficiencia de cada red y de cada interacción se evalúa en bloques separados, y el orden de estos bloques se aleatoriza (para evitar efectos sistemáticos atribuibles al orden de presentación), una muestra pequeña no se beneficiaría de este diseño. Así, diferencias reales entre las condiciones podrían no observarse en los resultados, a pesar de que los niveles elevados de glucosa en sangre (hiperglucemia) podrían estar teniendo un impacto negativo sobre el funcionamiento global del cerebro (Cox et al., 2005). Aunque estas alteraciones no impactan la precisión al responder (Patiño-Fernández et al., 2010), sí podrían evidenciarse en otro tipo de investigaciones donde se midan variables distintas (como las fisiológicas): los pacientes con DMT1 pueden ejecutar tareas cognitivas de forma similar a las personas sanas. Sin embargo, también se ha mostrado que las redes que se activan y de las cuales dependen ciertos procesos cognitivos son distintas en comparación con la población sana (e. g. Gallardo-Moreno et al., 2015). Estas diferencias pueden observarse a través de estudios fisiológicos o, como en nuestro caso, en los tiempos de respuesta que dependen de la eficiencia de la red de control ejecutivo.

Es necesario realizar una evaluación con un mayor número de participantes que aumente la probabilidad de detectar diferencias sutiles. Además, sería relevante estudiar la eficiencia de las redes atencionales en pacientes con DMT1 comparando las diferentes condiciones de glucemia presentes en el organismo: hiperglucemia, hipoglucemia y normoglucemia.

Una posible extensión a este estudio podría ser comparar los diferentes niveles de glucosa en sangre en pacientes con DM, mediante el uso de correlatos fisiológicos. Aunque en la literatura ya existen este tipo de análisis, sería importante estudiar los efectos de ambos extremos glucémicos sobre la eficiencia de las redes atencionales debido a que contamos con escasas investigaciones que evalúen la atención de forma particular. Además, no analizan dicha función con pruebas de alta especificidad, para tal fin, la evaluación de la atención través del Test de Redes Atencionales (Fan et al., 2002) sería una buena opción.

\section{Agradecimientos}

Este proyecto de investigación fue financiado por la Coordinación de Investigación Científica de la Universidad Michoacana de San Nicolás de Hidalgo, dentro de la convocatoria para apoyo a proyectos de investigación 2019.

\section{Referencias}

Asvold, B. O., Sand, T., Hestad, K., \& Bjorgaas, M. R. (2010). Cognitive Function in Type 1 Diabetic Adults with Early Exposure to Severe Hypoglycemia A 16-year follow-up study. Diabetes Care, 33(9), 1945-1947. doi: 10.2337/dc10-0621

Bade-White, P. A., \& Obrzut, J. E. (2009). The neurocognitive effects of type 1 diabetes mellitus in children and young adults with and without hypoglycemia. Journal of Developmental and Physical Disabilities, 21(5), 425-440. doi: 10.1007/s10882-009-9151-y

Brands, A. M., Biessels, G. J., De Haan, E. H., Kappelle, L. J., \& Kessels, R. P. (2005). The effects of type 1 diabetes on cognitive performance: A meta-analysis. Diabetes Care, 28(3), 726-735. doi: 10.2337/diacare.28.3.726

Brands, A. M., Biessels, G. J., Kappelle, L. J., De Haan, E. H., de Valk, H. W., Algra, A., \& Kessels, R. P. (2007). Cognitive functioning and brain MRI in patients with type 1 and type 2 diabetes mellitus: a comparative study. Dementia and Geriatric Cognitive Disorders, 23(5), 343-350. doi: 10.1159/000100980

Brands, A. M., Kessels, R. P., Hoogma, R. P., Henselmans, J. M., van der Beek Boter, J. W., Kappelle, L. J., ... Biessels, G. J. (2006). Cognitive performance, psychological well-being, and brain magnetic resonance imaging in older patients with type 1 diabetes. Diabetes, 55(6), 1800-1806. doi: $10.2337 / \mathrm{db} 05-1226$

Cato, A., \& Hershey, T. (2016). Cognition and Type 1 Diabetes in Children and Adolescents. Diabetes 
Manríquez-Calderón, S., et al. / RACC, 2020, Vol. 12, №3, 82-91

Spectrum, 29(4), 197-202. doi: 10.2337/ds16-0036

Cox, D. J., Kovatchev, B. P., Gonder-Frederick, L. A., Summers, K. H., McCall, A., Grimm, K. J., \& Clarke, W. L. (2005). Relationships between hyperglycemia and cognitive performance among adults with type 1 and type 2 diabetes. Diabetes Care, 28(1), 71-77. doi: 10.2337/diacare.28.1.71

Fan, J., McCandliss, B. D., Sommer, T., Raz, A., \& Posner, M. I. (2002). Testing the efficiency and independence of attentional networks. Journal of Cognitive Neuroscience, 14(3), 340-347. doi: $0.1162 / 089892902317361886$

Ferguson, S. C., Blane, A., Perros, P., McCrimmon, R. J., Best, J. J., Wardlaw, J., ... Frier, B. M. (2003). Cognitive ability and brain structure in type 1 diabetes relation to microangiopathy and preceding severe hypoglycemia. Diabetes, 52(1), 149-156. doi: 10.2337/diabetes.52.1.149

Ferguson, S. C., Blane, A., Wardlaw, J., Frier, B. M., Perros, P., McCrimmon, R. J., \& Deary, I. J. (2005). Influence of an early-onset age of type 1 diabetes on cerebral structure and cognitive function. Diabetes Care, 28(6), 1431-1437. doi: 10.2337/diacare.28.6.1431

Gallardo-Moreno, G. B., González-Garrido, A. A., Gudayol-Ferré, E., \& Guàrdia-Olmos, J. (2015). Type 1 diabetes modifies brain activation in young patients while performing visuospatial working memory tasks. Journal of Diabetes Research, 2015, 1-9. doi: 10.1155/2015/703512

Gaudieri, P. A., Chen, R., Greer, T. F., \& Holmes, C. S. (2008). Cognitive function in children with type 1 diabetes a meta-analysis. Diabetes Care, 31(9), 1892-1897. doi: $10.2337 / \mathrm{dc} 07-2132$

Hannonen, R., Tupola, S., Ahonen, T., \& Riikonen, R. (2003). Neurocognitive functioning in children with type-1 diabetes with and without episodes of severe hypoglycaemia. Developmental Medicine \& Child Neurology, 45(04), 262-268. doi: $10.1017 /$ S0012162203000501

Kirchhoff, B. A., Jundt, D. K., Doty, T., \& Hershey, T. (2017). A longitudinal investigation of cognitive function in children and adolescents with type 1 diabetes mellitus. Pediatric Diabetes, 18(6), 443449. doi: 10.1111 /pedi.12414

Kodl, C. T., \& Seaquist, E. R. (2008). Cognitive dysfunction and diabetes mellitus. Endocrine Reviews, 29(4), 494-511. doi: 10.1210/er.20070034

Li, W., Huang, E., \& Gao, S. (2017). Type 1 Diabetes Mellitus and Cognitive Impairments: A Systematic Review. Journal of Alzheimer's Disease, 57(1), 2936. doi: $10.3233 / J A D-161250$

Lin, A., Northam, E. A., Rankins, D., Werther, G. A., \& Cameron, F. J. (2010). Neuropsychological profiles of young people with type 1 diabetes $12 \mathrm{yr}$ after disease onset. Pediatric Diabetes, 11(4), 235-243. doi: 10.1111/j.1399-5448.2009.00588.x

Lin, A., Northam, E. A., Werther, G. A., \& Cameron, F. J. (2015). Risk factors for decline in IQ in youth with type 1 diabetes over the 12 years from diagnosis/illness onset. Diabetes Care, 38(2), 236242. doi: $10.2337 / \mathrm{dc} 14-1385$

MacLeod, J. W., Lawrence, M. A., McConnell, M. M., Eskes, G. A., Klein, R. M., \& Shore, D. I. (2010). Appraising the ANT: Psychometric and theoretical considerations of the Attention Network Test. Neuropsychology, 24(5), 637-651. doi: 10.1037/a0019803

Marzelli, M. J., Mazaika, P. K., Barnea-Goraly, N., Hershey, T., Tsalikian, E., Tamborlane, W., ... Reiss, A. L. (2014). Neuroanatomical correlates of dysglycemia in young children with type 1 diabetes. Diabetes, 63(1), 343-353. doi: $10.2337 / \mathrm{db} 13-0179$

McConnell, M. M., \& Shore, D. I. (2011). Mixing measures: testing an assumption of the Attention Network Test. Attention, Perception, \& Psychophysics, 73(4), 1096-1107. doi: 10.3758/s13414-010-0085-3

McCrimmon, R. J., Ryan, C. M., \& Frier, B. M. (2012). Diabetes and cognitive dysfunction. The Lancet, 379(9833), 2291-2299. doi: 10.1016/S01406736(12)60360-2

Monette, M. C., Baird, A., \& Jackson, D. L. (2014). A meta-analysis of cognitive functioning in nondemented adults with type 2 diabetes mellitus. Canadian Journal of Diabetes, 38(6), 401-408. doi: 10.1016/j.jcjd.2014.01.014

Naguib, J. M., Kulinskaya, E., Lomax, C. L., \& Garralda, M. E. (2009). Neuro-cognitive performance in children with type 1 diabetes a meta-analysis. Journal of Pediatric Psychology, 34(3), 271-282. doi: 10.1093/jpepsy/jsn074

Ohmann, S., Popow, C., Rami, B., König, M., Blaas, S., Fliri, C., \& Schober, E. (2010). Cognitive functions and glycemic control in children and adolescents with type 1 diabetes. Psychological Medicine, 4O(1), 95-103. doi: 10.1017/S0033291709005777

Organización Mundial de la Salud (2016). Nota Descriptiva No. 312. Recuperado de: http://www.who.int/mediacentre/factsheets/fs312/es 1

Patiño-Fernández, A. M., Delamater, A. M., Applegate, E. B., Brady, E., Eidson, M., Nemery, R., ... Richton, S. (2010). Neurocognitive functioning in preschool-age children with type 1 diabetes mellitus. Pediatric Diabetes, 11(6), 424-430. doi: 10.1111/j.1399-5448.2009.00618.x

Psychology Software Tools, Inc (2012). E-Prime 2.0 [software de computación]. Pittsburgh, PA: Psychology Software Tools, Inc.

R Core Team (2016). R: A language and environment for statistical computing [software de computación]. 
Manríquez-Calderón, S., et al. / RACC, 2020, Vol. 12, N³, 82-91

Austria, Viena: $\mathrm{R}$ Foundation For Statistical Computing.

Rodrigues, V. V., de Castro, R. M. A., Schamber, C. R., \& Bazotte, R. B. (2014). Hypoglycemia induced by insulin as a triggering factor of cognitive deficit in diabetic children. The Scientific World Journal, 2014, 1-9. doi: 10.1155/2014/616534

Ryan, C. M., Klein, B. E., Lee, K. E., Cruickshanks, K. J., \& Klein, R. (2016). Associations between recent severe hypoglycemia, retinal vessel diameters, and cognition in adults with type 1 diabetes. Journal of Diabetes and Its Complications, 30(8), 1513-1518. doi: 10.1016/j.jdiacomp.2016.08.010

Semenkovich, K., Patel, P. P., Pollock, A. B., Beach, K. A., Nelson, S., Masterson, J. J., ... Arbeláez, A. M. (2016). Academic abilities and glycaemic control in children and young people with Type 1 diabetes mellitus. Diabetic Medicine, 33(5), 668-673. doi: 10.1111/dme.12854

Wang, Y. F., Cui, Q., Liu, F., Huo, Y. J., Lu, F. M., Chen, H., \& Chen, H. F. (2014). A new method for computing attention network scores and relationships between attention networks. PloS One, 9(3), 1-8. doi: 10.1371/journal.pone.0089733

Wang, Y. F., Jing, X. J., Liu, F., Li, M. L., Long, Z. L., Yan, J. H., \& Chen, H. F. (2015). Reliable attention network scores and mutually inhibited inter-network relationships revealed by mixed design and nonorthogonal method. Scientific Reports, 5(1), 1-10. doi: $10.1038 /$ srep10251

Wessels, A. M., Rombouts, S. A. R. B., Remijnse, P. L., Boom, Y., Scheltens, P., Barkhof, F., ... \& Snoek, F. J. (2007). Cognitive performance in type 1 diabetes patients is associated with cerebral white matter volume. Diabetología, 50(8), 1763-1769. doi: 10.1007/s00125-007-0714-0

Westlye, L. T., Grydeland, H., Walhovd, K. B., \& Fjell, A. M. (2010). Associations between regional cortical thickness and attentional networks as measured by the attention network test. Cerebral Cortex, 21(2), 345-356. doi: 10.1093/cercor/bhq101 